\title{
ELECTROENCEPHALOGRAPHIC PARAMETERS IN CHILDREN WITH OUTCOMES OF THE PERINATAL CEREBRAL NERVOUS SYSTEM DAMAGES
}

\author{
S. Shamansurov, N. Sohieva, C. Uzakova, N. Ziyamuhamedova \\ Child Neurology, Tashkent Institute of Postgraduate Medical Education, Tashkent, Uzbekistan
}

Background and aims: Pathology of the central nervous system in children is one of the actual problems of the modern neurology. To study electroencephalographic parameters in children with retardation of the psychomotor and verbal development.

Methods: We have studied 60 children at the age from 3 months to 3 years. The patients were divided into 2 groups:

group 1 included children with retardation of psychomotor development (30 children),

group 2 comprised of children with disturbances of verbal development (30 children).

The results were analyzed with use of visual and quantitative evaluation of EEG.

Results: The results showed that in children from group 1 there were prevailed delta-brushes in $12(40 \%)$, frontal acute waves in $10(33,3 \%)$ and diffusive slow activity in $10(33,3 \%)$ children; and in children group 2 the changes of low voltage indifferential activity was prevailed in $10(33,3 \%)$ children and delta-brushes - in $9(30,0 \%)$. All these changes showed retardation of the maturation and myelinization of the corticalsubcortical structures of brain. Thus, the results of evaluation showed that more deep changes were registered on EEG in children with retardation of psychomotor development.

Conclusions: The early performance of electroencephalographic investigations allows timely diagnosis of the retardation of the psychomotor and verbal development in children that will promote prevention of the long-term outcomes of the perinatal damages of the nervous system and improvement of the social adaptation of the children population. 